\title{
Everything Has a Cause: Leveraging Causal Inference in Legal Text Analysis
}

\author{
Xiao Liu ${ }^{1 *}$ Da Yin ${ }^{2 *}$ Yansong Feng ${ }^{1,3}$, Yuting $\mathbf{W u}^{1}$ and Dongyan Zhao ${ }^{1,3}$ \\ ${ }^{1}$ Wangxuan Institute of Computer Technology, Peking University, China \\ ${ }^{2}$ Computer Science Department, University of California, Los Angeles \\ ${ }^{3}$ The MOE Key Laboratory of Computational Linguistics, Peking University, China \\ \{lxlisa, fengyansong, wyting, zhaody\} @pku.edu.cn \\ da.yin@cs.ucla.edu
}

\begin{abstract}
Causal inference is the process of capturing cause-effect relationship among variables. Most existing works focus on dealing with structured data, while mining causal relationship among factors from unstructured data, like text, has been less examined, but is of great importance, especially in the legal domain. In this paper, we propose a novel Graph-based Causal Inference $(G C I)$ framework, which builds causal graphs from fact descriptions without much human involvement and enables causal inference to facilitate legal practitioners to make proper decisions. We evaluate the framework on a challenging similar charge disambiguation task. Experimental results show that GCI can capture the nuance from fact descriptions among multiple confusing charges and provide explainable discrimination, especially in few-shot settings. We also observe that the causal knowledge contained in $G C I$ can be effectively injected into powerful neural networks for better performance and interpretability. Code and data are available at https://github.com/xxxiaol/GCI/.
\end{abstract}

\section{Introduction}

Causal inference is the process of exploring how changes on variable $T$ affect another variable $Y$. Here we call $T$ and $Y$ as treatment and outcome, respectively, and the changes on $T$ are called intervention. In other words, the process of drawing a conclusion about whether and how $Y$ changes when intervening on $T$ is called causal inference.

Most research in causal inference is devoted to analyzing structured data. Take the research question how smoking causes lung cancer (Pearl and Mackenzie, 2018) as an example. Smoking, lung cancer, together with distractors like age are extracted from structured data, like electronic health records, and considered as factors. Usually, such

\footnotetext{
* Equal contribution.

Corresponding author.
}

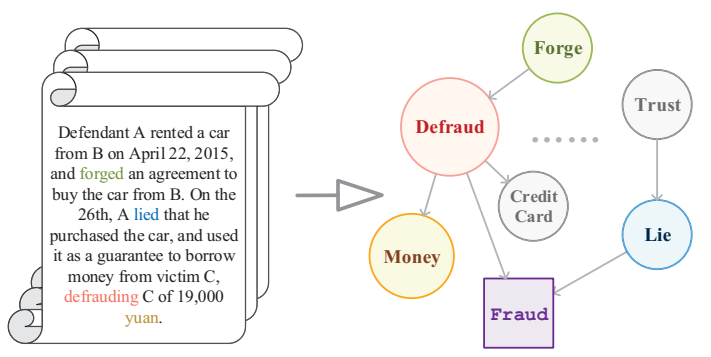

Figure 1: An example of generated causal graph for the charge fraud. Colored words are matched between the exemplified fact description and the graph.

studies properly organize those factors into humandesigned structures, e.g., a causal directed acyclic graph (Wright, 1921) with factors \{smoking, age, lung cancer $\}$ as nodes and causal relations $\{$ smoking $\rightarrow$ lung cancer, age $\rightarrow$ lung cancer $\}$ as edges, and perform inference on such structures.

Recent works attempt to integrate text information into causal inference (Egami et al., 2018; Veitch et al., 2019; Yao et al., 2019; Keith et al., 2020), but they mainly treat the text as a single node in the causal graph, which is relatively coarsegrained. For instance, Yao et al. (2019) investigate how the complaints from consumers affect the company's responses (admission or denial). They regard the entire text of complaint as a treatment, without looking into different aspects of the text, like the events that consumers complained about and the compensation that consumers requested.

Actually, discovering causal relationship inside unstructured and high-dimensional data, like text, is also beneficial or even crucial for scenarios involving reading comprehensive text and making decisions accordingly. For instance, when a legal AI system assists judges to deal with complicated cases that involve multiple parties and complex events, causal inference could help to figure out the exact distinguishable elements that are crucial for fair and impartial judgements. As shown in Figure 1, if the system can automatically spot two 
essential key points, 1) the deceitful acts of the defendant, and 2) obtaining properties from the victim, from the unstructured fact descriptions, then the prediction fraud can be more convincing and helpful, rather than a label from a black box. In practice, we would expect a legal AI system to provide human-readable and sound explanations to help the court make the right decisions. It is worthwhile especially for underdeveloped areas, where such techniques could help the judges of rural areas with more trustworthy references from previous judgements. This would further help to maintain the principle of treating like cases alike in many continental law systems. A main challenge in judicial practice is to distinguish between similar charges, we thus propose a new task similar charge disambiguation as a testbed. Cases of similar charges often share similar context, and a system is expected to mine the nuance of reasoning process from the text.

However, performing causal inference on fact descriptions of criminal cases is not trivial at all. It poses the following challenges. 1) Without expert involvement, it is not easy to extract factors that are key to prediction, and organize them in a reasonable form that can both facilitate the inference process and tolerate noise. For example, automatically extracted elements may not cover all the key points in the textual descriptions, and automatically built graphs may contain unreliable edges. 2) It is not easy to benefit from both traditional causal inference models and modern neural architectures.

In this paper, we propose a novel Graph-based Causal Inference $(G C I)$ framework, which could effectively apply causal inference to legal text analysis. $G C I$ first recognizes key factors by extracting keywords from the fact descriptions and clustering similar ones into groups as individual nodes. Then we build causal graphs on these nodes with a causal discovery algorithm that can tolerate unobserved variables, reducing the impact of missing elements. We further estimate the causal strength of each edge, to weaken unreliable edges as much as possible, and apply the refined graph to help decision making. Experimental results show that our GCI framework can induce reasonable causal graphs and capture the nuance from plain text for legal applications, especially with few training data.

We also explore the potential of GCI by integrating the captured causal knowledge into neu- ral network (NN) models. We propose two approaches: 1) imposing the causal strength constraints to the NN's attention weights; 2) applying recurrent neural networks on the causal chains extracted from our causal graphs. Experiments indicate that our methods can successfully inject the extracted causal knowledge and thus enhance the NN models. We also show that integrating $G C I$ helps to mitigate the underlying bias in data towards the final prediction.

Our main contributions are as follows: 1) We propose a novel graph-based causal inference $(G C I)$ framework to apply causal inference to unstructured text, without much human involvement. 2) We explore to equip popular neural network models with our $G C I$, by encouraging neural models to learn from causal knowledge derived from $G C I .3)$ We evaluate our methods on a legal text analysis task, similar charge disambiguation, and experimental results show that our GCI can capture the nuance from plain fact descriptions, and further help improve neural models with interpretability.

\section{Background}

Two types of questions are typically related to causality. The first is whether there is causal relationship between a set of variables, and the second question is when two variables $T$ and $Y$ are causally related, how much would $Y$ change if we change the value of $T$. Both of them are discussed in our GCI framework, and we first briefly introduce the key concepts.

\subsection{Causal Discovery}

Causal discovery corresponds to the first type of questions. From the view of graph, causal discovery requires models to infer causal graphs from observational data. In our GCI framework, we leverage Greedy Fast Causal Inference (GFCI) algorithm (Ogarrio et al., 2016) to implement causal discovery. GFCI combines score-based and constraintbased algorithms. It merges the best of both worlds, performing well like score-based methods, and not making too unrealistic assumptions like constraintbased ones. Specifically, GFCI does not rely on the assumption of no latent confounders, thus is suitable in our situation. More details of GFCI algorithm and its advantage is provided in Appendix A.

The output of GFCI is a graphical object called Partial Ancestral Graph (PAG). PAG is a mixed graph containing the features common to all Di- 


\begin{tabular}{c|c}
\hline Edge & Meaning \\
\hline $\mathrm{A} \rightarrow \mathrm{B}$ & A causes B. \\
$\mathrm{A} \leftrightarrow \mathrm{B}$ & There is an unobserved confounder of A and B. \\
$\mathrm{A} \circ \rightarrow \mathrm{B}$ & Either A causes B, or unobserved confounder. \\
$\mathrm{A} \circ-\circ \mathrm{B}$ & Either A causes B, or B causes A, or unobserved confounder. \\
\hline
\end{tabular}

Table 1: Summary of edge types in PAG.

rected Acyclic Graphs (DAGs) that represent the same conditional independence relationship over the measured variables. In other words, PAG entails all the possibilities of valid DAGs concerning the original data. In causal inference settings (Zhang, 2008), four types of edges are provided in PAG, as listed in Table 1. With PAG, we are able to consider unobserved confounders and the uncertainty in causal inference.

\subsection{Causal Strength Estimation}

Causal strength estimation deals with the second type of questions. It is the very task to quantify the causal strength of each learned relation, i.e., whether the relation is strong or weak. To precisely estimate causal strength, confounders need to be kept the same. Confounder is a variable causally influencing both treatment $T$ and outcome $Y$. Take the example of smoking, lung cancer and age in Section 1 . Here we study if there is causal relationship between smoking $(T)$ and lung cancer $(Y)$, and age is a confounder $C$. It is straightforward to compare the proportion of lung cancer among smokers and non-smokers. However, age influences both smoking and lung cancer. Older people are more likely to smoke. They also have a much higher risk of suffering from cancer. If we do not consider the value of age, its influence to lung cancer will be regarded as smoking's influence, thus wrongly amplify the causal effect of smoking to lung cancer.

In our GCI framework, we apply Average Treatment Effect (ATE) (Holland, 1986) as a measure of causal strength. All variables are binary in our work. So given an edge $T \rightarrow Y$, we quantify how the outcome $Y$ is expected to change if we modify the treatment $T$ from 0 to 1 :

$$
\psi_{T, Y}=E[Y \mid d o(T=1)]-E[Y \mid d o(T=0)],
$$

where $E$ means expectation, and the do-calculus $d o(T=1)$ indicates intervention on $T$, setting its value to 1 .

We utilize the Propensity Score Matching (PSM) method to estimate ATE. PSM finds the pairs of comparable samples with the most similar propensity scores, where each pair consists of one sample in the treated group and one in the untreated. Given the great similarity between the two samples, we could make a direct comparison between them. Specifically, propensity score $L(z)=P(T=1 \mid Z=z)$ is the probability of treatment being assigned to 1 given a set of observed confounders $z$. As $T$ is binary, we have $T \Perp Z \mid L(\Perp$ means independence). So matching on propensity scores equals matching on the full set of confounders.

\section{Graph-based Causal Inference Framework}

Our graph-based causal inference (GCI) framework consists of three parts, constructing the causal graph, estimating causal strength on it, and making decisions. Figure 2 shows the overall architecture.

\subsection{Task Definition}

We first define the similar charge disambiguation task. Given the fact descriptions of criminal cases $\mathbb{D}=\left\{d_{1}, d_{2}, \ldots, d_{N}\right\}$, a system is expected to classify each case into one charge from the similar charge set $\mathbb{C}=\left\{c_{1}, c_{2}, \ldots, c_{M}\right\}$.

\subsection{Causal Graph Construction}

Extracting Factors. To prepare nodes for the causal graph, we calculate the importance of word $w_{j}$ for charge $c_{i}$ using YAKE (Campos et al., 2020). We enhance YAKE with inverse document frequency (Jones, 1972) to extract more discriminative words of each charge.

To discriminate the similar charges, we select $p$ words with the highest importance scores for each charge, cluster them into $q$ classes to merge similar keywords. The $q$ classes together with the $M$ charges form the nodes of the causal graph. All these factors are binary. When the graph is applied to a case, each factor is of value 1 if it exists in this case, and 0 if not. Unlike factors extracted by experts, automatically extracted keywords may be incomplete, resulting in unobserved confounders in causal discovery.

Learning Causal Relationship. The next step is to build edges for the graph, in other words, discover the causal relationship between different factors. To learn causal relations and tackle the unobserved confounder problem, we use GFCI (Ogarrio et al., 2016), which does not rely on the assumption 


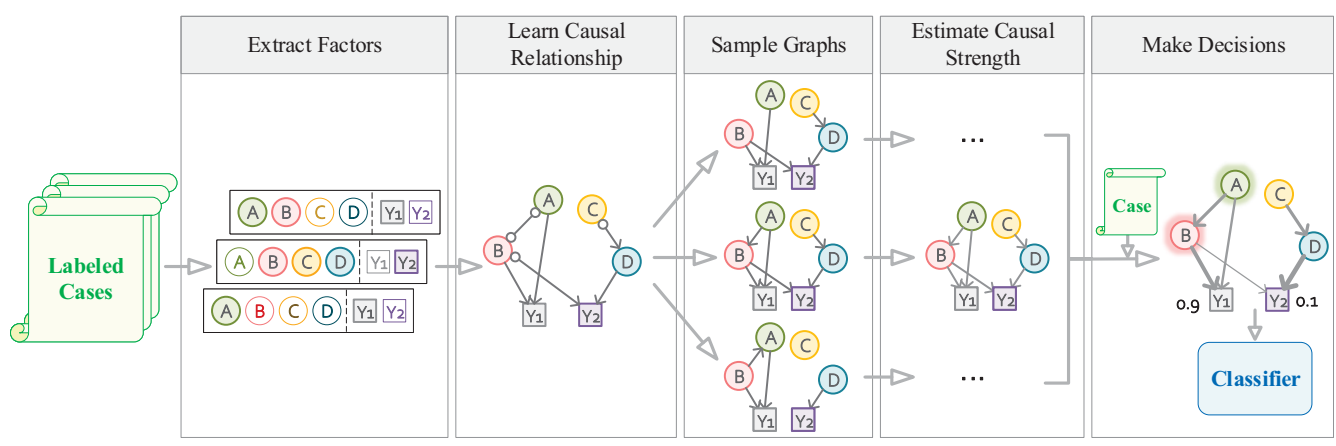

Figure 2: Overall architecture of GCI. In the Extract Factors phase, solid circles indicate that these factors exist in this case, while hollow circles mean the opposite. For the nodes of causal graphs, $A, B, C$, and $D$ denote the key points contributing to prediction, while $Y_{1}$ and $Y_{2}$ indicate the charges to discriminate. Four types of edges $\rightarrow$, $\leftrightarrow, \circ \rightarrow$, and $\circ-\circ$ exist in the PAG in the Learn Causal Relationship phase, and they are converted to $\rightarrow$ edges in the sampled graphs. In the rightmost phase, shaded factors are matched between fact descriptions of cases and the graph.

of no unobserved confounders. As mentioned in Section 2, the output of GFCI is a PAG. Appendix $\mathrm{C}$ gives an example of a generated PAG in detail.

We further introduce constraints to filter noisy edges. First, as the judgement is made based on the fact description, we do not allow edges from charge nodes to other ones, e.g., an edge from fraud to lie is prohibited. Second, given that causes usually appear before effects in time (Black, 1956), and fact descriptions in legal text are often written in the temporal order of events, we thus consider the chronological order of descriptions as temporal constraints to filter noisy edges. If factor $A$ appears after $B$ in most cases, we will not allow the edge from $A$ to $B$. Note that this constraint does not imply there is an edge from $B$ to $A$, as chronological order is not a sufficient condition of causality.

Sampling Causal Graphs. PAG contains uncertain relations shown in Table 1, which leaves challenges for quantification and further application. So we sample $Q$ causal graphs from PAG. Among the four edge types, $\rightarrow$ and $\leftrightarrow$ are clear: in each sampled graph, $\rightarrow$ edges are retained and $\leftrightarrow$ edges are removed (because they do not indicate causal relations between the two nodes). For $\circ \rightarrow$ edges, they have two possible choices: being kept (cause) and being removed (unobserved confounder). In the absence of true possibility, we simply keep an edge with $1 / 2$ probability, and remove it with another $1 / 2$. And for $0 \circ$ edges, we give $1 / 3$ probability for $\rightarrow, \leftarrow$, and no edge, respectively. The quality of each sampled graph $G_{q}$ is measured by its fitness with data $\mathbf{X}$, where we use the Bayesian information criterion $\operatorname{BIC}\left(G_{q}, \mathbf{X}\right)$ to estimate (Schwarz et al., 1978).

\subsection{Strength Estimation on Causal Graphs}

As the resulting graphs are noisy in nature, we estimate the strength of the learned causal relations to refine a sampled causal graph. We assign high strength to edges with strong causal effect, and near-zero strength to edges that do not indicate causal relations or with weak effect. We regard the Average Treatment Effect $\psi_{T, Y}^{G}$ (ATE, Section 2.2) as the strength of $T \rightarrow Y$ in graph $G$, and utilize the Propensity Score Matching (PSM, Section 2.2) to measure it:

$$
\hat{\psi}_{T, Y}^{G}=\left[\sum_{i: t_{i}=1}\left(y_{i}-y_{j}\right)+\sum_{i: t_{i}=0}\left(y_{j}-y_{i}\right)\right] / N,
$$

where $j=\underset{k: t_{k} \neq t_{i}}{\operatorname{argmin}}\left|L\left(z_{i}\right)-L\left(z_{k}\right)\right|$ means the most similar instance in the opposite group of $i$, and $t_{i}, y_{i}, z_{i}$ are the value of treatment, outcome and confounders of instance $i$, respectively.

\subsection{Making Decisions}

When applying the sampled causal graphs to the similar charge disambiguation task, we simply extract factors and map the case description with the graph accordingly, and decide which charge in $\mathbb{C}$ is more appropriate to this case. Firstly, we compute the overall causal strength of each factor $T_{j}$ to $Y_{i}$ among the $Q$ sampled causal graphs, where $Y_{i}$ represents whether charge $c_{i}$ is committed:

$$
\tilde{\psi}_{T_{j}, Y_{i}}=\sum_{q=1}^{Q} \operatorname{BIC}\left(G_{q}, \mathbf{X}\right) \times \hat{\psi}_{T_{j}, Y_{i}}^{G_{q}},
$$


where $\hat{\psi}_{T_{j}, Y_{i}}^{G_{q}}$ is the measured causal strength in $G_{q}$, and is 0 if edge $T_{j} \rightarrow Y_{i}$ does not exist in $G_{q}$.

For each case, we then map the text with the graphs, and calculate scores for each charge:

$$
S\left(Y_{i}\right)=\sum_{T_{j} \in \operatorname{Tr}\left(Y_{i}\right)} \tilde{\psi}_{T_{j}, Y_{i}} \times \tau\left(T_{j}\right), i \in\{1, \ldots, M\},
$$

where $\tau\left(T_{j}\right)$ is a dummy variable indicating the presence of $T_{j}$ in this case, and $\operatorname{Tr}\left(Y_{i}\right)$ is the set of treatments of $Y_{i}$ (from the view of graph, the nodes pointing to $Y_{i}$ ). The calculated scores are fed into a random forest classifier $(\mathrm{Ho}, 1995)$ to learn thresholds between the charges. More advanced classifiers can also be used.

\section{Integration of Causal Analysis and Neural Networks}

Neural networks (NN) are considered to be good at exploring large volumes of textual data. This motivates us to integrate the causal framework with NN, to benefit each other. Here we propose two integration methods as shown in Figure 3.

\subsection{Imposing Strength Constraint}

First, we inject the estimated causal strength to constrain the attention weights of a Bi-LSTM with attention model (Zhou et al., 2016). A Bi-LSTM layer is first applied to the fact descriptions to obtain contextual embeddings $\mathbf{H}=$ $\left\{\mathbf{h}_{1}, \mathbf{h}_{2}, \ldots, \mathbf{h}_{n}\right\}, \mathbf{h}_{i} \in \mathbb{R}^{b_{0}}$, where $b_{0}$ is the dimension of embeddings. Then, an attention layer assigns different weights $\left\{a_{1}, a_{2}, \ldots, a_{n}\right\}$ to each word, and sums the words up according to the weights to build a text embedding $\mathbf{v}$ :

$$
a_{i}=\frac{\exp \left(\mathbf{q}^{T} \cdot \mathbf{h}_{i}\right)}{\sum_{k=1}^{n} \exp \left(\mathbf{q}^{T} \cdot \mathbf{h}_{k}\right)}, \mathbf{v}=\sum_{i=1}^{n} a_{i} \times \mathbf{h}_{i},
$$

where $\mathbf{q} \in \mathbb{R}^{b_{0}}$ is a learnable query vector. Finally, we apply two fully connected layers to the text embedding $\mathbf{v}$, and form the prediction vector $\mathbf{r}_{\text {cons }}$.

Besides a cross-entropy loss $L_{\text {cross }}$ on $\mathbf{r}_{\text {cons }}$, we introduce an auxiliary loss $L_{\text {cons }}$ to guide the attention module with the causal strength learned from $G C I$. Given the golden label $c_{j}$, for each word $w_{i}$ which belongs to the factor $f, \tilde{\psi}_{T_{f}, Y_{j}}$ is the corresponding causal strength, and $g_{i}$ is the normalized strength over the whole sequence. $L_{\text {cons }}$ is set to make the attention weights close to the normalized

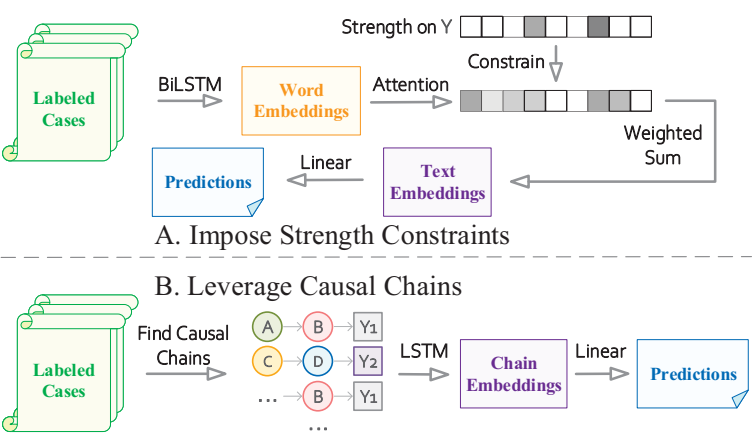

Figure 3: Two ways of integrating causal analysis and neural networks.

strength:

$$
\begin{aligned}
& L_{\text {cons }}=\sum_{i=1}^{n}\left(a_{i}-g_{i}\right)^{2}, \\
& L=L_{\text {cross }}+\alpha L_{\text {cons }} .
\end{aligned}
$$

Note that in the validation and testing stages, the inputs do not contain any strength constraint and golden charge information. Therefore, we select the epoch with the least cross-entropy loss in the validation stage to evaluate on the test set.

\subsection{Leveraging Causal Chains}

Causal chains are another type of knowledge that can be captured from causal graphs. In the legal scenario, causal chains depict the process of committing crimes. They can also be treated as the summarization of cases or behavioural patterns for each charge. Therefore, the second approach is to leverage the causal chains directly, as the chains may contain valuable information for judgement. For a given text, we extract factors and traverse all causal chains composed by the factors from the sampled causal graphs, Chains $=$ $\left\{\right.$ chain $_{1}$, chain $_{2}, \ldots$, chain $\left._{m}\right\}$. In this task, we only consider chains ending up with treatments of charges, as they are more relevant with the judgement. An LSTM layer is applied to each chain, and all the chains are pooled to build case representation $\mathbf{c} \in \mathbb{R}^{b_{0}}$ :

$$
\begin{gathered}
\mathbf{c h}_{i}=\sum_{j=1}^{l_{i}}\left(\operatorname{LSTM}\left(\operatorname{chain}_{i}\right)_{j}\right), \\
\mathbf{c}=\operatorname{MaxPooling}\left(\operatorname{BIC}\left(G_{q}, \mathbf{X}\right) \times \mathbf{c h}_{i}\right), \\
1 \leq i \leq m, \operatorname{chain}_{i} \in G_{q},
\end{gathered}
$$

where $l_{i}$ indicates the length of $\operatorname{chain}_{i}$. The case representation $\mathbf{c}$ is then fed to two fully connected layers to make the prediction $\mathbf{r}_{\text {chain }}$, and a crossentropy loss is used to optimize the model. 


\begin{tabular}{l|c|c}
\hline Charge Sets & Charges & \#Cases \\
\hline Personal Injury & $\begin{array}{c}\text { Intentional Injury \& Murder \& } \\
\text { Involuntary Manslaughter }\end{array}$ & $\begin{array}{c}6377 / 2282 / \\
1989\end{array}$ \\
\hline Violent Acquisition & $\begin{array}{c}\text { Robbery \& Seizure \& } \\
\text { Kidnapping }\end{array}$ & $\begin{array}{c}5020 / 2113 / \\
622\end{array}$ \\
\hline F\&E & Fraud \& Extortion & $3536 / 2149$ \\
\hline E\&MPF & $\begin{array}{c}\text { Embezzlement \& } \\
\text { Misappropriation of Public Funds }\end{array}$ & $2391 / 1998$ \\
\hline AP\&DD & $\begin{array}{c}\text { Abuse of Power \& } \\
\text { Dereliction of Duty }\end{array}$ & $1950 / 1938$ \\
\hline
\end{tabular}

Table 2: Summary of the similar charge sets.

\section{Experiments and Evaluation}

\subsection{Experimental Setup}

Dataset. For the similar charge disambiguation task, we pick five similar charge sets from the Criminal Law of the People's Republic of China (Congress, 2017), which are hard to discriminate in practice (Ouyang et al., 1999), and select the corresponding fact descriptions from the Chinese AI and Law Challenge (CAIL2018) (Xiao et al., 2018). Detailed statistics of the charge sets are given in Table 2. Note we filter out the cases whose judgements include multiple charges from one charge set. The fact descriptions in our dataset are in Chinese.

Our Models. We evaluate our graph-based causal inference $(G C I)$ framework as described in Section 3, and two models integrating $G C I$ with NN (Bi-LSTM+Att+Cons and CausalChain) as described in Section 4.

Comparison Models. To study the effect of causal relationship captured by $G C I$, we implement a variant called GCI-co, which is built upon a correlation-based graph rather than our discovered causal graph. In detail, we compute the Pearson correlation coefficient $\phi$ for every two factors, and draw an edge if $\phi>0.5$. The direction of the edge is from the factor that appears earlier in the text more often, to the other. Then we compare GCI and two integration methods with $\mathrm{NN}$ baselines, including LSTM, Bi-LSTM and Bi-LSTM+Att. Bi$L S T M+A t t$ is a common backbone of legal judgement prediction models, while we do not add multitask learning (Luo et al., 2017) and expert knowledge (Xu et al., 2020) for simplicity. Since the prior knowledge learned from pre-trained models may result in unfair comparison, we do not choose the models such as BERT (Devlin et al., 2018) as baselines and backbones to eliminate the influence.
Previous works integrating text into causal inference are not able to find causal relationships inside text, so we do not take them into comparison.

We select a set of training ratios, $1 \%, 5 \%, 10 \%$, $30 \%$, and $50 \%$, to study how the performance gap changes along with different training data available. For each setting, we run the experiments on three random seeds and report the average accuracy (Acc) and macro-F1 (F1). More details about baselines, parameter selection, and training process are in Appendix B.

\subsection{Main Results}

Table 3 reports the charge disambiguation performance of our models and comparison models.

Causal Graph vs. Correlation-based Graph. GCI outperforms GCI-co by $4.5 \%$ on average Acc, and $9.8 \%$ on average F1, indicating the graph constructed by mining causal relations better captures the relationship between charges and factors.

Causal Inference vs. Neural Networks. Comparing GCI with NN baselines LSTM, Bi-LSTM and Bi-LSTM+Att, we observe in few-shot settings $(1 \%, 5 \%), G C I$ outperforms NNs by about $10 \%$ on average, since NNs tend to underfit in few-shot settings. However, with the increase of training data, the performance gap becomes narrower and consequently, NNs outperform $G C I$ in several cases. Compared with GCI, NNs have the advantage of learning from large amounts of unstructured data.

Adding Strength Constraints. We can see that Bi-LSTM +Att+Cons outperforms Bi-LSTM+Att by around $1-5 \%$. The performance gap is much larger in few-shot settings. This suggests that our estimated causal strength is helpful for attention-based models to capture the key information in the text.

Causal Chains vs. Whole Text. Both CausalChain and LSTM are a straightforward application of unidirectional LSTM, but over different texts, one for our extracted causal chains and the other for the whole fact description. We find CausalChain outperforms LSTM by $8.2 \%$ on average Acc and $11.7 \%$ on average F1. The difference shows that causal chains contain condensed key information that contributes to the judgement, while the whole description may contain far more irrelevant information that may disturb the prediction. We also conduct experiments on combining causal chains and the 


\begin{tabular}{|c|c|c|c|c|c|c|c|}
\hline Models & & $\begin{array}{l}\text { Personal } \\
\text { Injury }\end{array}$ & $\begin{array}{c}\text { Violent } \\
\text { Acquisition }\end{array}$ & $\mathbf{F} \& \mathbf{E}$ & E\&MPF & AP\&DD & Average \\
\hline \multirow{5}{*}{ LSTM } & $1 \%$ & $60.94 / 37.91$ & $58.48 / 29.33$ & $63.91 / 47.00$ & $53.56 / 39.84$ & $52.08 / 46.13$ & $57.79 / 40.04$ \\
\hline & $5 \%$ & $61.97 / 44.88$ & $67.09 / 35.86$ & $71.60 / 68.68$ & $59.89 / 56.88$ & $54.12 / 48.53$ & $62.93 / 50.97$ \\
\hline & $10 \%$ & $76.45 / 67.81$ & $65.64 / 47.62$ & $82.14 / 80.74$ & $70.21 / 70.00$ & $55.46 / 51.29$ & $69.98 / 63.49$ \\
\hline & $30 \%$ & $85.37 / 81.27$ & $74.43 / 66.05$ & $88.10 / 87.33$ & $71.60 / 70.82$ & $65.61 / 65.19$ & $77.02 / 74.13$ \\
\hline & $50 \%$ & 85.67 / 83.02 & $80.10 / 72.27$ & $90.04 / 89.06$ & $75.59 / 75.46$ & 69.65 / 69.62 & $80.21 / 77.89$ \\
\hline \multirow{5}{*}{ Bi-LSTM } & $1 \%$ & $62.29 / 40.81$ & $53.86 / 33.25$ & $62.95 / 43.27$ & $54.54 / 41.91$ & $48.98 / 37.84$ & $56.52 / 39.42$ \\
\hline & $5 \%$ & $74.00 / 69.52$ & $65.18 / 38.99$ & $60.34 / 56.96$ & $61.88 / 61.63$ & $51.77 / 46.23$ & $62.63 / 54.66$ \\
\hline & $10 \%$ & $76.66 / 71.86$ & $67.10 / 46.07$ & $85.31 / 84.37$ & $60.08 / 53.34$ & $60.20 / 57.95$ & $69.87 / 62.72$ \\
\hline & $30 \%$ & $85.46 / 82.53$ & $75.30 / 64.12$ & $87.57 / 86.58$ & $70.45 / 69.64$ & $65.45 / 65.12$ & $76.85 / 73.60$ \\
\hline & $50 \%$ & $87.19 / 85.01$ & 78.43 / 69.94 & $90.43 / 89.83$ & $76.08 / 75.78$ & $71.12 / 70.50$ & $80.65 / 78.21$ \\
\hline \multirow{5}{*}{ GCI } & $1 \%$ & $69.54 / 49.77$ & $57.08 / 42.55$ & $82.81^{*} / 82.56^{*}$ & $74.65^{*} / 70.22^{*}$ & 62.47 / 61.72 & $69.31^{*} / 61.36^{*}$ \\
\hline & $5 \%$ & $81.19 / 75.58$ & $69.70 / \mathbf{6 0 . 3 9}^{\dagger}$ & $88.25 / \mathbf{8 7 . 2 4}{ }^{\dagger}$ & $\mathbf{8 3 . 2 7}^{\dagger} / \mathbf{8 3 . 0 6}^{\dagger}$ & $\mathbf{7 8 . 0 9}^{\dagger} / \mathbf{7 7 . 9 5}^{\dagger}$ & $\mathbf{8 0 . 1 0}^{\dagger} / \mathbf{7 6 . 8 4}^{\dagger}$ \\
\hline & $10 \%$ & $80.33 / 74.50$ & $74.06 / \mathbf{6 7 . 3 1} 1^{\S}$ & 87.97 / 87.51 & $85.23^{\S} / 84.62^{\S}$ & $\mathbf{7 8 . 3 6} 6^{\S} / \mathbf{7 8 . 3 1} 1^{\S}$ & $81.19^{\S} / 78.45^{\S}$ \\
\hline & $30 \%$ & $84.83 / 80.10$ & $75.99 / 70.64$ & $89.31 / 88.39$ & $88.55^{\ddagger} / 88.21^{\ddagger}$ & $80.82 / \mathbf{8 0 . 5 6}^{\ddagger}$ & $83.90^{\ddagger} / 8^{1.58} 8^{\ddagger}$ \\
\hline & $50 \%$ & $85.72 / 81.62$ & $76.31 / 71.45$ & $90.41 / 89.14$ & $89.01^{\natural} / 88.63^{\natural}$ & $81.01^{\natural} / 80.90^{\natural}$ & 84.49 / 82.35 \\
\hline \multirow{5}{*}{ GCI-co } & $1 \%$ & $67.49 / 44.43$ & 63.70* / 34.64 & $75.72 / 67.60$ & $69.08 / 67.20$ & $64.93^{*} / 64.41^{*}$ & $68.19 / 55.66$ \\
\hline & $5 \%$ & $76.70 / 63.94$ & $67.65 / 34.35$ & $86.63 / 85.81$ & $82.23 / 81.86$ & 73.94 / 73.77 & 77.43 / 67.95 \\
\hline & $10 \%$ & $68.05 / 45.37$ & $69.26 / 46.39$ & $85.62 / 84.41$ & $81.23 / 79.64$ & $74.21 / 74.05$ & $75.67 / 65.97$ \\
\hline & $30 \%$ & $77.31 / 63.45$ & $70.42 / 50.94$ & $81.44 / 80.54$ & $85.71 / 85.20$ & $74.43 / 74.28$ & $77.86 / 70.88$ \\
\hline & $50 \%$ & $79.21 / 69.37$ & $70.38 / 50.78$ & $79.30 / 77.58$ & $84.39 / 83.72$ & $74.16 / 73.99$ & $77.49 / 71.09$ \\
\hline \multirow{5}{*}{ CausalChain } & $1 \%$ & 73.20* / 60.31* & $63.60 / 44.02 *$ & $68.01 / 52.93$ & $66.97 / 56.66$ & $63.13 / 62.30$ & $66.98 / 55.24$ \\
\hline & $5 \%$ & $\mathbf{8 1 . 9 9}^{\dagger} / \mathbf{7 6 . 0 3}^{\dagger}$ & $70.57 / 59.85$ & $\mathbf{8 8 . 6 4}^{\dagger} / 87.21$ & $75.13 / 74.74$ & $71.75 / 70.38$ & $77.62 / 73.64$ \\
\hline & $10 \%$ & $81.21 / 74.71$ & 73.50 / 66.66 & 87.59 / 86.36 & $79.75 / 79.45$ & $74.43 / 74.11$ & $79.30 / 76.26$ \\
\hline & $30 \%$ & $85.61 / 81.00$ & $74.93 / 67.30$ & $89.10 / 88.19$ & $81.63 / 81.25$ & $\mathbf{8 0 . 9 0}^{\ddagger} / 80.50$ & $82.43 / 79.65$ \\
\hline & $50 \%$ & $86.41 / 83.11$ & 75.66 / 68.47 & $90.45 / 89.21$ & $81.25 / 80.09$ & $80.03 / 79.89$ & $82.76 / 80.16$ \\
\hline \multirow{5}{*}{ Bi-LSTM+Att } & $1 \%$ & $62.16 / 41.70$ & $58.21 / 32.97$ & $67.99 / 62.80$ & $57.90 / 50.67$ & $53.20 / 41.78$ & $59.89 / 45.99$ \\
\hline & $5 \%$ & $78.29 / 72.81$ & $67.50 / 50.68$ & $85.30 / 84.28$ & $61.86 / 55.38$ & $58.76 / 53.03$ & 70.34 / 63.23 \\
\hline & $10 \%$ & $81.51 / 78.36$ & $67.97 / 58.26$ & $88.07 / 87.33$ & $75.38 / 74.86$ & $58.82 / 55.82$ & $74.35 / 70.93$ \\
\hline & $30 \%$ & $86.07 / 83.49$ & $80.47 / 72.55$ & 88.97 / 88.41 & $81.53 / 81.14$ & $72.84 / 72.65$ & $81.98 / 79.65$ \\
\hline & $50 \%$ & $87.25 / 85.38$ & $82.27 / 74.15$ & $91.56 / 91.05$ & $82.29 / 82.11$ & $73.70 / 73.65$ & $83.41 / 81.27$ \\
\hline \multirow{5}{*}{$\begin{array}{c}\text { Bi-LSTM+Att } \\
+ \text { Cons }\end{array}$} & $1 \%$ & $70.12 / 59.46$ & $54.29 / 40.34$ & $78.25 / 76.80$ & $61.03 / 60.62$ & $53.84 / 44.93$ & $63.51 / 56.43$ \\
\hline & $5 \%$ & $79.07 / 75.89$ & $\mathbf{7 3 . 0 9} \mathbf{9}^{\dagger} / 56.84$ & 86.80 / 86.35 & $66.86 / 59.89$ & $72.27 / 72.18$ & $75.62 / 70.23$ \\
\hline & $10 \%$ & $\mathbf{8 3 . 3 3}^{\S} / \mathbf{7 9 . 7 0}^{\S}$ & $\mathbf{7 6 . 2 6}^{\S} / 64.62$ & $\mathbf{8 8 . 7 6}^{\S} / \mathbf{8 8 . 0 2}^{\S}$ & $80.03 / 79.64$ & $73.53 / 73.48$ & $80.38 / 77.09$ \\
\hline & $30 \%$ & $86.55^{\ddagger} / 83.85^{\ddagger}$ & $81.48^{\ddagger} / 73.15^{\ddagger}$ & $\mathbf{8 9 . 8 0}^{\ddagger} / \mathbf{8 9 . 3 5}^{\ddagger}$ & 81.82 / 81.31 & $79.46 / 79.35$ & 83.82 / 81.40 \\
\hline & $50 \%$ & $88.31^{\natural} / 86.18^{\natural}$ & $82.72^{\natural} / 76.03^{\natural}$ & $92.05^{\natural} / 91.55^{\natural}$ & 83.02 / 82.69 & $80.72 / 80.64$ & $85.36^{\natural} / 83.42^{\natural}$ \\
\hline
\end{tabular}

Table 3: Performance on similar charge disambiguation. The first number is Acc and the second number is F1. Highest results are in bold, and different symbols indicate different training ratios.

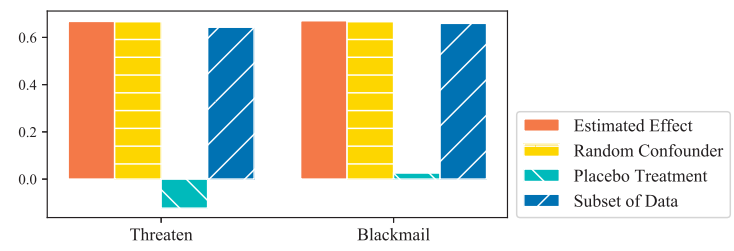

Figure 4: An example of sensitivity analysis for treatments of extortion in the causal graph of F\&E.

whole plain text, but simply concatenating them does not work well since the whole text may introduce noise, and better integration methods are needed, which we leave for future work.

\section{Analysis}

\subsection{Quality of Causal Graphs}

To analyze the robustness of the causal discovery process, we apply sensitivity analysis on the causal graphs. In detail, we make disturbance to the original causal relations, and examine the sensitivity of causal effect towards the violations. Following Kiciman and Sharma (2018), we use three re- futers for examination: 1) Random Confounder, a new confounder with random value is added to the graph, and ideally, the causal strength should remain the same as before. 2) Placebo Treatment, the value of a treatment is replaced by a random value, so the treatment becomes a placebo, and the strength should be zero. 3) Subset of Data, we use a subset of cases to recalculate the strength. Ideally, the strength estimation will not vary significantly.

We take a sampled causal graph of F\&E (Fraud \& Extortion) as an example, and exhibit the refuters on the treatments of charge extortion in Figure 4. Causal strength is almost the same as before after Random Confounder and Subset of Data refutation; and turns to nearly zero after Placebo Treatment. The results show that our graph construction method is robust against disturbance.

\subsection{Causal Chains in Graph}

Causal chains manifest how effective GCI is in terms of inducing common patterns of suspect's 


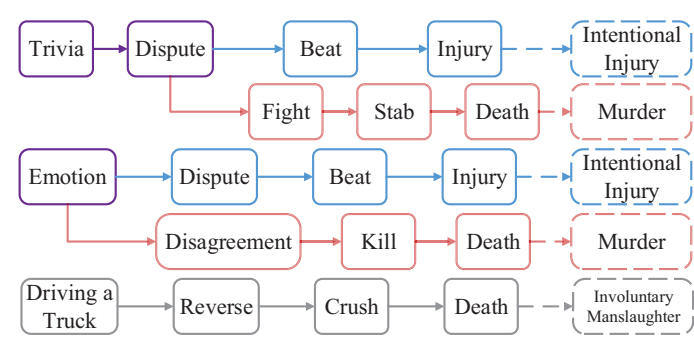

Figure 5: Causal chains in Personal Injury's graph.

behaviours. It is helpful for people to better understand the core part of legal cases. Here we select the causal graph of Personal Injury (Intentional Injury \& Murder \& Involuntary Manslaughter) and showcase several causal chains underlying the text. As shown in Figure 5, the chains depict common patterns of these charges, from the initial causes, to the final criminal behaviour. More examples are provided in Appendix D.

Now the question is how the graph structures help to discriminate similar charges. Here we analyze the nuance between the causal chains of two similar charges E\&MPF (Embezzlement \& Misappropriation of Public Funds). For both charges, the cases often first give the background that someone held a certain position of authority, thus had power. Then both kinds of cases describe that the person illegally obtained a large amount of money by utilizing his power. While the cases in E\&MPF share very similar context, there exists nuance between them: embezzlement emphasizes that someone privately and illegally possesses the bulk of money, while misappropriation of public funds emphasizes that someone would temporally use the money for a certain purpose. By observing the causal chains of two charges, $G C I$ could capture the slight difference well: for embezzlement, the causal chains tend to be work / take charge of $\rightarrow$ take advantage of (position/power); for misappropriation of public funds, the causal chains tend to be take charge of $\rightarrow$ take advantage of (position/power) $\rightarrow$ misappropriate $\rightarrow$ profit. The difference between the former and latter chains is whether the person had subsequent behaviour (e.g., using the money for purposes like making profits). We could observe that the difference in causal chains accords with the definitions of the two charges.

\subsection{Effect of Integrating Causal Strength with Attention}

Following Lei et al. (2017), we conduct human evaluation on words accorded with high attention

\begin{tabular}{l|c|c}
\hline Charge Sets & Bi-LSTM+Att & Bi-LSTM+Att+Cons \\
\hline Personal Injury & 3.03 & $\mathbf{3 . 1 7}$ \\
Violent Acquisition & 3.18 & $\mathbf{3 . 7 4}$ \\
F\&E & 3.34 & $\mathbf{3 . 6 5}$ \\
E\&MPF & 3.13 & $\mathbf{3 . 2 7}$ \\
AP\&DD & 3.08 & $\mathbf{3 . 1 3}$ \\
\hline
\end{tabular}

Table 4: Results of human evaluation. Better results are in bold.

weights and compare the evaluation results of standard $(B i-L S T M+A t t)$ and constraint-based attention models (Bi-LSTM+Att+Cons).

For each set of charges, we train both models with $10 \%$ data, and randomly select 30 cases that both models predict correctly. For the total 150 cases, we showcase content, charge names, attention weights above 0.05 , and corresponding words. Each participant is asked to score from 1 to 5 for the extent of how beneficial the extracted keywords are to disambiguation. A higher score means that the attention weights indeed capture the keywords. Each case is assigned to at least four participants. Results are shown in Table 4. We observe that the constraint-based model is better at explanation than normal attention-based models on all five charge groups. Take Violent Acquisition as an example. Although the cases are predicted correctly by $B i$ $L S T M+A t t$, the model tends to attend to words bag, $R M B$ and value, which frequently occur but cannot be treated as clues for judgement. Instead, $B i$ $L S T M+A t t+C o n s$ values factors such as grab, rob and hold, which are more helpful for judgement.

\section{Related Works}

Causal Inference with Text. Recently, a few works try to take text into account when performing causal inference. Landeiro and Culotta (2016) introduce causal inference to text classification, and manage to remove bias from certain out-of-text confounders. Wood-Doughty et al. (2018) use text as a supplement of missing data and measurement error for the causal graphs constructed by structured data. Egami et al. (2018) focus on mapping text to a low-dimensional representation of the treatment or outcome. Veitch et al. (2019) and Yao et al. (2019) treat text as confounder and covariate, which help to make causal estimation more accurate. These works all build causal graphs manually, and regard text as a whole to be one of the factors. In contrast, we set our sights on text containing rich causal information in itself. Paul (2017) looks into text by computing propensity score for each word, but only 
focuses on causal relationship between words and sentiment. We instead take a causal graph perspective, discover and utilize causal relationship inside text to perform reasoning.

Neural Networks for Causal Discovery. Recently, researchers attempt to apply neural networks to causal discovery (Ponti and Korhonen, 2017; Alvarez-Melis and Jaakkola, 2017; Ning et al., 2018; Gao et al., 2019; Weber et al., 2020). However, Alvarez-Melis and Jaakkola (2017) model causal relationship by correlation, which may introduce bias into causal inference; Ning et al. (2018) and Gao et al. (2019) merely focus on capturing causality by explicit textual features or supervision from labeled causal pairs. There are also a line of works focusing on how to use neural networks to summarize confounders and estimate treatment effects (Louizos et al., 2017; Yao et al., 2018; Künzel et al., 2018), which are parts of the whole causal inference process. Weber et al. (2020) show how to formalize causal relationships in script learning, but it is limited to pairwise learning of events and cannot be generalized to sequential and compositional events.

Legal Judgement Prediction. Previous works in legal text analysis focus on the task of legal judgement prediction (LJP). Luo et al. (2017) and Zhong et al. (2018) exploit neural networks to solve LJP tasks. Zhong et al. (2020) provide interpretable judgements by iteratively questioning and answering. Another line pays attention to confusing charges: Hu et al. (2018) manually design discriminative attributes, and $\mathrm{Xu}$ et al. (2020) use attention mechanisms to highlight differences between similar charges. Using knowledge derived from causal graphs, $G C I$ exhibits a different and interpretable discrimination process.

\section{Discussion}

Although GCI is effective on its own and when working with powerful neural network models, there is still room for further improvement.

More Precise Causal Inference Models. The causal estimation results of $G C I$ is based on the constructed causal graphs in former stages, and the automated construction process may bring imprecise factors and even omissions. As clustering algorithms try to summarize the general characteristics of text, descriptions with subtle differences may be clustered into one factor, but the differences matter in legal judgement. For example, in Personal Injury's graph, different ways of killing are summarized as the factor kill, therefore lose valuable information. Specifically, beaten to death might occur in cases of involuntary manslaughter, while shooting cases are more likely to be associated with murder. Also, factors with low frequency may be omitted in clustering, but are actually useful for discrimination. Overall, under the circumstance without much expert effort, it is worthwhile to explore how to construct a more reliable causal graph.

Deep understanding on legal documents. Although $G C I$ to some extent tackles the challenges of incapability of causal inference methods on unstructured text, it may make mistakes when facing complex fact descriptions. Negation semantics is a typical example. It is occasional to see negation word usage in fact descriptions, which usually indicates that someone did not have a certain behaviour. However, GCI has not considered this aspect, and may be awry in cases containing negation usage. Besides, pronoun resolution is also an important aspect that may confuse models. For example, certain behaviour is done by the victim and the subject of the behaviour is a pronoun. If the model was unaware of the subject of the behaviour, it would be counted as criminal's behaviour and introduces noise to later inference stage. Moreover, intent could be a decisive factor when discriminating charges, for example, murder and involuntary manslaughter. But it may not be mentioned explicitly in the fact descriptions. It should be better to recover the complete course of fact and recognize the implicit intents between the lines with deep understanding of the context and relevant common sense knowledge.

\section{Conclusions}

We propose $G C I$, a graph-based causal inference framework to discover causal information in text, and design approaches to integrate causal models and neural networks. In the similar charge disambiguation task, we show our approaches capture important evidence for judgement and nuance among charges. Further analysis demonstrates the quality of causal graphs, value of causal chains, and interpretability of computed causal strength. 


\section{Ethical Considerations}

\subsection{Intended Use}

We aim to facilitate legal service with our proposed $G C I$, providing valuable evidence instead of directly making judgements. We hope that legal AI models could assist legal workers in underdeveloped areas, helping them to explore key points in cases, discriminate from similar crimes, and make better decisions. By treating like cases alike in different regions of the country, influences of judges' intellectual flaws and arbitrariness are weakened, and rights of people, both the defendants and the victims, are protected.

Failure Mode. The model may give wrong evidence in some cases, but this will not cause significantly bad impact. The process of GCI is transparent. Extracted factors, the causal relations between them, and causal chains that lead to the final decision are all shown to users. By checking these "rationales" of model reasoning, users can clearly find where goes wrong, and not adopt the outputs, or intervene and correct the model.

Misuse Potential. We emphasize that such a model cannot be used individually, as the trial process is seriously conducted and regulated by the judicial system. In the actual judicial process, the prosecutors, judges, and lawyers are under strict supervision. We do not think there is a possibility for them to misuse computer models.

\subsection{Bias Analysis}

Criminal behaviour is very unbalanced in gender. Take the three charges in our Personal Injury charge set as an example. Lin and Zou (2020) counted gender ratio in criminal cases from 2013 to 2017 in China, and the ratios of male defendant are $94.70 \%$ (Intentional Injury), 87.72\% (Murder), and 93.97\% (Involuntary Manslaughter). The disparity in defendants of male and female leads to the small proportion of female cases in training corpus. Therefore, female cases may be inadequately trained. If this results in more incorrect predictions for female cases, women's rights are violated.

Following Dixon et al. (2018) and Park et al. (2018), we use False Positive Equality Difference (FPED) and False Negative Equality Difference (FNED) to examine the performance difference in

\begin{tabular}{l|c|c}
\hline Metrics & Bi-LSTM+Att & Bi-LSTM+Att+Cons \\
\hline FPED & 0.048 & $\mathbf{0 . 0 3 2}$ \\
FNED & 0.065 & $\mathbf{0 . 0 4 9}$ \\
\hline
\end{tabular}

Table 5: Results of equality difference. Better results are in bold.

two genders. They are defined as:

$$
\begin{aligned}
& \mathrm{FPED}=\sum_{t \in T}\left|\mathrm{FPR}-\mathrm{FPR}_{t}\right|, \\
& \mathrm{FNED}=\sum_{t \in T}\left|\mathrm{FNR}-\mathrm{FNR}_{t}\right|,
\end{aligned}
$$

where FPR is false positive rate of classification, FNR is false negative rate, and $T=$ $\{$ male, female $\}$. The two metrics quantify the extent of variation between the performances of two genders.

Applying them to Bi-LSTM+Att and Bi$L S T M+A t t+C o n s$ models in Personal Injury charge set, the results are shown in Table 5. The model with causal constraints achieves smaller variance measured by both metrics, which reduce between $1 / 4$ to $1 / 3$ of the unfairness in performance of $B i-L S T M+A t t$. This shows the superiority of our model with causal knowledge. Compared with normal neural networks, our constraint-based model utilizes causal relations, which are more stable to the number of occurrences.

Though adding causal knowledge narrows the equality difference, it still exists in $\mathrm{Bi}$ LSTM+Att+Cons (the metrics are greater than zero). Other types of bias may also exist in our model, given that the training corpora contain decisions of humans and systemic bias of humans may be preserved. Further debiasing method is needed if the model is put into real use.

In general, we believe that adding causal knowledge to decision making will help debiasing, and the transparent exhibition of causal graphs and chains will enable people to find biases in time and correct them.

\section{Acknowledgments}

This work is supported in part by National Hi-Tech R\&D Program of China (2018YFC0831900). We would like to thank the anonymous reviewers for the helpful discussions and suggestions. Also, we would thank Yuxuan Lai, Liunian Harold Li, Jieyu Zhao, Chen Wu and Xingchen Lan for advice about 
experiments and writing. For any correspondence, please contact Yansong Feng.

\section{References}

David Alvarez-Melis and Tommi Jaakkola. 2017. A causal framework for explaining the predictions of black-box sequence-to-sequence models. In Proceedings of the 2017 Conference on Empirical Methods in Natural Language Processing, pages 412421, Copenhagen, Denmark. Association for Computational Linguistics.

Max Black. 1956. Why cannot an effect precede its cause? Analysis, 16(3):49-58.

Ricardo Campos, Vítor Mangaravite, Arian Pasquali, Alípio Jorge, Célia Nunes, and Adam Jatowt. 2020. Yake! keyword extraction from single documents using multiple local features. Information Sciences, 509:257-289.

David Maxwell Chickering. 2002. Optimal structure identification with greedy search. Journal of machine learning research, 3(Nov):507-554.

National People's Congress. 2017. Criminal Law of the People's Republic of China. China Legal Publishing House.

Jacob Devlin, Ming-Wei Chang, Kenton Lee, and Kristina Toutanova. 2018. Bert: Pre-training of deep bidirectional transformers for language understanding. arXiv preprint arXiv:1810.04805.

Lucas Dixon, John Li, Jeffrey Sorensen, Nithum Thain, and Lucy Vasserman. 2018. Measuring and mitigating unintended bias in text classification. In Proceedings of the 2018 AAAI/ACM Conference on AI, Ethics, and Society, pages 67-73.

Naoki Egami, Christian J Fong, Justin Grimmer, Margaret E Roberts, and Brandon M Stewart. 2018. How to make causal inferences using texts. arXiv preprint arXiv:1802.02163.

Lei Gao, Prafulla Kumar Choubey, and Ruihong Huang. 2019. Modeling document-level causal structures for event causal relation identification. In Proceedings of the 2019 Conference of the North American Chapter of the Association for Computational Linguistics: Human Language Technologies, Volume 1 (Long and Short Papers), pages 1808-1817, Minneapolis, Minnesota. Association for Computational Linguistics.

Tin Kam Ho. 1995. Random decision forests. In Proceedings of 3rd international conference on document analysis and recognition, volume 1, pages 278282. IEEE.

Paul W Holland. 1986. Statistics and causal inference. Journal of the American statistical Association, 81(396):945-960.
Zikun Hu, Xiang Li, Cunchao Tu, Zhiyuan Liu, and Maosong Sun. 2018. Few-shot charge prediction with discriminative legal attributes. In Proceedings of the 27th International Conference on Computational Linguistics, pages 487-498.

Karen Sparck Jones. 1972. A statistical interpretation of term specificity and its application in retrieval. Journal of documentation.

Katherine A Keith, David Jensen, and Brendan O'Connor. 2020. Text and causal inference: A review of using text to remove confounding from causal estimates. arXiv preprint arXiv:2005.00649.

Emre Kiciman and Amit Sharma. 2018. Tutorial on causal inference and counterfactual reasoning. In ACM KDD International Conference on Knowledge Discovery and Data Mining.

Sören R. Künzel, Bradly C. Stadie, Nikita Vemuri, Varsha Ramakrishnan, Jasjeet S. Sekhon, and Pieter Abbeel. 2018. Transfer learning for estimating causal effects using neural networks. CoRR, abs/1808.07804.

Virgile Landeiro and Aron Culotta. 2016. Robust text classification in the presence of confounding bias. In Thirtieth AAAI Conference on Artificial Intelligence.

Tao Lei et al. 2017. Interpretable neural models for natural language processing. Ph.D. thesis, Massachusetts Institute of Technology.

Wei Lin and Shaokun Zou. 2020. The Bluebook on the Big Data of Criminal Justice. Peking University Press.

Christos Louizos, Uri Shalit, Joris M. Mooij, David A. Sontag, Richard S. Zemel, and Max Welling. 2017. Causal effect inference with deep latent-variable models. In Advances in Neural Information Processing Systems 30: Annual Conference on Neural Information Processing Systems 2017, 4-9 December 2017, Long Beach, CA, USA, pages 6446-6456.

Bingfeng Luo, Yansong Feng, Jianbo Xu, Xiang Zhang, and Dongyan Zhao. 2017. Learning to predict charges for criminal cases with legal basis. In Proceedings of the 2017 Conference on Empirical Methods in Natural Language Processing, pages 2727 2736, Copenhagen, Denmark. Association for Computational Linguistics.

James MacQueen et al. 1967. Some methods for classification and analysis of multivariate observations. In Proceedings of the fifth Berkeley symposium on mathematical statistics and probability, volume 1, pages 281-297. Oakland, CA, USA.

Qiang Ning, Zhili Feng, Hao Wu, and Dan Roth. 2018. Joint reasoning for temporal and causal relations. In Proceedings of the 56th Annual Meeting of the Association for Computational Linguistics (Volume 1: Long Papers), pages 2278-2288, Melbourne, Australia. Association for Computational Linguistics. 
Juan Miguel Ogarrio, Peter Spirtes, and Joe Ramsey. 2016. A hybrid causal search algorithm for latent variable models. In Conference on Probabilistic Graphical Models, pages 368-379.

Tao Ouyang, Kejia Wei, and Renwen Liu. 1999. Confusing crimes, noncrime, and boundaries between crimes. Chinese People's Public Security University Press.

Ji Ho Park, Jamin Shin, and Pascale Fung. 2018. Reducing gender bias in abusive language detection. arXiv preprint arXiv:1808.07231.

Michael Paul. 2017. Feature selection as causal inference: Experiments with text classification. In Proceedings of the 21st Conference on Computational Natural Language Learning (CoNLL 2017), pages 163-172.

Judea Pearl and Dana Mackenzie. 2018. The book of why: the new science of cause and effect. Basic Books.

Edoardo Maria Ponti and Anna Korhonen. 2017. Event-related features in feedforward neural networks contribute to identifying causal relations in discourse. In Proceedings of the 2 nd Workshop on Linking Models of Lexical, Sentential and Discourselevel Semantics, pages 25-30, Valencia, Spain. Association for Computational Linguistics.

Joseph Ramsey, Madelyn Glymour, Ruben SanchezRomero, and Clark Glymour. 2016. A million variables and more: the fast greedy equivalence search algorithm for learning high-dimensional graphical causal models, with an application to functional magnetic resonance images. International Journal of Data Science and Analytics, 2(3):121-129.

Gideon Schwarz et al. 1978. Estimating the dimension of a model. The annals of statistics, 6(2):461-464.

Yan Song, Shuming Shi, Jing Li, and Haisong Zhang. 2018. Directional skip-gram: Explicitly distinguishing left and right context for word embeddings. In Proceedings of the 2018 Conference of the North American Chapter of the Association for Computational Linguistics: Human Language Technologies, Volume 2 (Short Papers), pages 175-180.

Peter L Spirtes, Christopher Meek, and Thomas S Richardson. 2013. Causal inference in the presence of latent variables and selection bias. arXiv preprint arXiv:1302.4983.

Victor Veitch, Dhanya Sridhar, and David M Blei. 2019. Using text embeddings for causal inference. arXiv preprint arXiv:1905.12741.

Noah Weber, Rachel Rudinger, and Benjamin Van Durme. 2020. Causal inference of script knowledge. ArXiv, abs/2004.01174.
Zach Wood-Doughty, Ilya Shpitser, and Mark Dredze. 2018. Challenges of using text classifiers for causal inference. In Proceedings of the Conference on Empirical Methods in Natural Language Processing. Conference on Empirical Methods in Natural Language Processing, volume 2018, page 4586. NIH Public Access.

S. Wright. 1921. Correlation and causation. Journal of Agricultural Research, 20:557-585.

Chaojun Xiao, Haoxi Zhong, Zhipeng Guo, Cunchao Tu, Zhiyuan Liu, Maosong Sun, Yansong Feng, Xianpei Han, Zhen Hu, Heng Wang, et al. 2018. Cail2018: A large-scale legal dataset for judgment prediction. arXiv preprint arXiv:1807.02478.

Nuo Xu, Pinghui Wang, Long Chen, Li Pan, Xiaoyan Wang, and Junzhou Zhao. 2020. Distinguish confusing law articles for legal judgment prediction. arXiv preprint arXiv:2004.02557.

Liuyi Yao, Sheng Li, Yaliang Li, Mengdi Huai, Jing Gao, and Aidong Zhang. 2018. Representation learning for treatment effect estimation from observational data. In Advances in Neural Information Processing Systems 31: Annual Conference on Neural Information Processing Systems 2018, NeurIPS 2018, 3-8 December 2018, Montréal, Canada, pages 2638-2648.

Liuyi Yao, Sheng Li, Yaliang Li, Hongfei Xue, Jing Gao, and Aidong Zhang. 2019. On the estimation of treatment effect with text covariates. In Proceedings of the 28th International Joint Conference on Artificial Intelligence, pages 4106-4113. AAAI Press.

Jiji Zhang. 2008. Causal reasoning with ancestral graphs. Journal of Machine Learning Research, 9(Jul):1437-1474.

Haoxi Zhong, Zhipeng Guo, Cunchao Tu, Chaojun Xiao, Zhiyuan Liu, and Maosong Sun. 2018. Legal judgment prediction via topological learning. In Proceedings of the 2018 Conference on Empirical Methods in Natural Language Processing, pages 3540-3549.

Haoxi Zhong, Yuzhong Wang, Cunchao Tu, Tianyang Zhang, Zhiyuan Liu, and Maosong Sun. 2020. Iteratively questioning and answering for interpretable legal judgment prediction. $A A A I$.

Peng Zhou, Wei Shi, Jun Tian, Zhenyu Qi, Bingchen Li, Hongwei Hao, and Bo Xu. 2016. Attention-based bidirectional long short-term memory networks for relation classification. In Proceedings of the 54th annual meeting of the association for computational linguistics (volume 2: Short papers), pages 207212. 


\section{A GFCI Algorithm}

GFCI (Ogarrio et al., 2016) is a combination of a constraint-based causal discovery algorithm FCI (Spirtes et al., 2013) and a score-based algorithm FGES (Ramsey et al., 2016). Based on the skeleton of FCI, it uses initialization from FGES to improve accuracy and efficiency.

FCI takes sample data and optional background knowledge as input, and guarantees to represent the Markov equivalence class of the true causal DAG. It has two phases: adjacency and orientation. It does not rely on no latent confounder assumption, but it performs relatively poorly, especially on real data.

On the other hand, FGES greedily searches over potential DAGs, and outputs the highest scoring graph it finds. It is fast and accurate when its assumptions are satisfied, but it relies on the condition that there are no latent confounders.

GFCI takes the output of FGES as an initialized graph, and the graph is further augmented by FCI's adjacency phase, in which some adjacencies are removed by conditional independence tests. A similar process happens in the orientation phase. Orientations of FGES are provided as initialization, and further orientations from FCI are applied. Proof in Ogarrio et al. (2016) guarantees the GFCI algorithm outputs a PAG that represents the true causal DAG.

Figure 6 shows the advantage of GFCI. When factor $A$ is unobserved, score-based algorithms like GES (Chickering, 2002) will wrongly discover edge $B \rightarrow D$, while GFCI correctly recognizes the relation between factors $B$ and $D$ : there is an unobserved confounder of $B$ and $D$.

\section{B Implementation Details}

When the training set ratio is $1 \%$, we select $p=15$ keywords for each charge, and cluster the keywords of both charges into $q=20$ factors; for the training set ratio $5 \%$ and $10 \%, p=25$ and $q=30$; for the training set ratio $30 \%$ and $50 \%, p=40$ and $q=60$. We use K-means (MacQueen et al., 1967) for clustering. When learning causal relationship, besides cases in the training set, we also use some unlabeled cases of other charges to improve the interpretability of the causal graph. Note that removing these cases will not hurt the performance. We sample $Q=5$ causal graphs for each PAG.

For the neural network based models (both the baselines and our proposed models), $10 \%$ of the

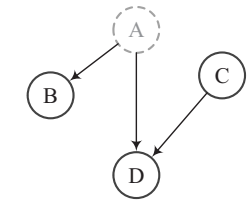

(a) True causal graph

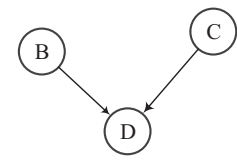

(b) GES output

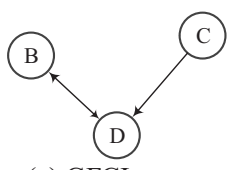

(c) GFCI output
Figure 6: Comparison between the output of GES and GFCI. $A$ is a latent variable, which is also an unobserved confounder of $B$ and $D$. This implies that in fact there is no causal relationship between $B$ and $D$ but without the consideration of unobserved confounders, GES tends to be awry in this situation.

training set is used as the validation set. The models are trained for 30 epochs, and the early stopping mechanism takes effect when the validation loss does not drop for more than 1000 batches. We use Adam optimizer with learning rate 0.001 , and the dropout rate is 0.5 . We use Tencent AILab Chinese Word Embedding (Song et al., 2018) as word embeddings, and the dimension of each word is 200 . The hidden sizes are $b_{0}=128$ and $b_{1}=64$. The batchsize is selected from $\{4,8,16,32,64,128\}$ considering the dataset size and training set ratio. For Bi-LSTM+Att+Cons, $\alpha$ is manually tuned within $\{0.1,0.25,0.5,1\}$, selecting the one with best validation F1. All the hyperparameters are empirically selected and kept the same for different models in the same dataset and the same training set ratio.

To reduce the impact of data imbalance between charges, we apply data augmentation to smaller charges whose cases are 3 times fewer than the biggest charge in that set, regardless of the training ratio. We did not keep them 1:1, in order to reflect their distributions in the real world.

The models are trained on Intel Xeon CPU. It takes about one hour to construct the causal graphs, and a few minutes to perform inference.

\section{Example of Generated PAG}

In this section, we further show part of a Partial Ancestor Graph (PAG) generated by our GCI framework. The example is shown in Figure 7.

For the edge between Hold People and Hostage, the arrow is $\rightarrow$, indicating there is a causal relationship between the factors Hold People and Hostage (the person who was held might become a hostage).

For the edge between Hold Knife and Hold People, the arrow is $\circ \rightarrow$, which means there might be an unobserved confounder which causes the two factors, and there might exist causal relationship 


\begin{tabular}{|c|c|}
\hline Charge Sets & Chains \\
\hline Personal Injury & $\begin{array}{l}\text { trivia } \rightarrow \text { dispute } \rightarrow \text { beat } \rightarrow \text { injury } \rightarrow \text { intentional injury } \\
\text { emotion } \rightarrow \text { disagreement } \rightarrow \text { kill } \rightarrow \text { death } \rightarrow \text { murder } \\
\text { driving a truck } \rightarrow \text { reverse } \rightarrow \text { crush } \rightarrow \text { death } \rightarrow \text { involuntary manslaughter }\end{array}$ \\
\hline Violent Acquisition & $\begin{array}{l}\text { hold a knife } \rightarrow \text { violence } \rightarrow \text { cash } \rightarrow \text { robbery } \\
\text { necklace } \rightarrow \text { pull } \rightarrow \text { seizure } \\
\text { hostage } \rightarrow \text { threaten } \rightarrow \text { ask for } \rightarrow \text { ransom } \rightarrow \text { kidnapping }\end{array}$ \\
\hline F\&E & $\begin{array}{l}\text { approach } \rightarrow \text { trust } \rightarrow \text { lie } \rightarrow \text { fraud } \\
\text { forge } \rightarrow \text { defraud } \rightarrow \text { fraud } \\
\text { relationship } \rightarrow \text { nude photos } \rightarrow \text { threaten } \rightarrow \text { extortion }\end{array}$ \\
\hline E\&MPF & $\begin{array}{l}\text { work } \rightarrow \text { take advantage of (position/power) } \rightarrow \text { embezzlement } \\
\text { falsely report } \rightarrow \text { arbitrage } \rightarrow \text { embezzlement } \\
\text { take charge of } \rightarrow \text { take advantage of (position/power) } \rightarrow \text { misappropriate } \rightarrow \text { profit } \\
\rightarrow \text { misappropriation of public funds }\end{array}$ \\
\hline AP\&DD & $\begin{array}{l}\text { (use) position } \rightarrow \text { (provide) convenience } \rightarrow \text { abuse of power } \\
\text { complete understand } \rightarrow \text { violate } \rightarrow \text { abuse of power } \\
\text { ignore } \rightarrow \text { result in (bad things) } \rightarrow \text { dereliction of duty }\end{array}$ \\
\hline
\end{tabular}

Table 6: Exhibition of causal chains for each charge set.

between the two factors. In the first possible situation, the confounder could be Latent1, the intent to commit a crime. A person who intends to commit a crime may hold a knife and hold people. And in the second possible situation, the person who holds a knife is likely to hold people. Therefore, there is uncertainty in predicting the causal relationship.

For the edge between Hostage and Family, the arrow is $\longleftrightarrow$, which means there is an unobserved confounder which causes the two factors, and there does not exist causal relationship between them. For example, the confounder could be Latent2, the intent to obtain properties from the hostage. The intent could not only make the suspect grab the hostage's money, but also contact families to ask for ransom.

\section{Exhibition of More Causal Chains}

Table 6 showcases three chains for each charge set. They depict common patterns of behaviours of defendants that are charged with the crime at the tail of the chains. Note that each chain merely describes one possibility and one aspect of the criminal behaviour, and the chain itself may not be sufficient to initiate such a lawsuit. For example, the chain complete understand $\rightarrow$ violate $\rightarrow$ abuse of power of charge set AP\&DD shows that the litigant deliberately violated the rules, but he/she will be charged with abuse of power only when his/her action results in major loss of public property.

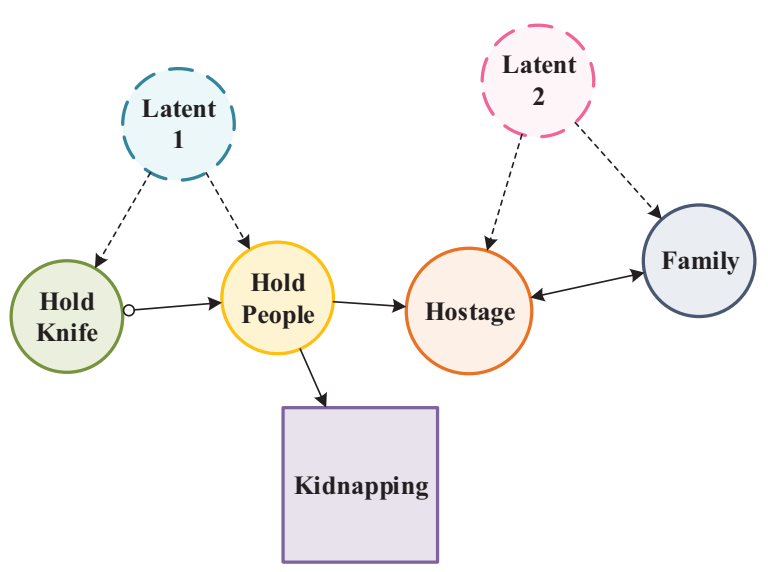

Figure 7: An example of part of the generated PAG of charge kidnapping in charge set Violent Acquisition. Latent1 and Latent 2 are unobserved variables that do not exist in the generated PAG. Detailedly, Latent 1 may denote the suspect's intent to commit a crime; and Latent 2 may denote the suspect's intent to obtain properties from the hostage. 